\title{
Development of a Photosynthesis Model with an Emphasis on Ecological Applications
}

\author{
III. Carbon Dioxide and Oxygen Dependencies
}

\author{
J.D. Tenhunen, J.A. Weber, L.H. Filipek, and D.M. Gates
}

The Biological Station, Division of Biological Sciences, The University of Michigan, Ann Arbor, MI 48109, USA

\begin{abstract}
Summary. A theoretical description of the simultaneous processes of photosynthesis and photorespiration in a single leaf is developed, based on the hypothesis that carbon dioxide and oxygen compete for the active site of ribulose diphosphate carboxylase. Michaelis-Menten kinetics and competitive inhibition at the end of a diffusion path provide the basic structure of the model. Data of Ludwig (1972) from sunflower are analyzed according to the formulation. This description is part of a more general physiologicalecological model of photosynthesis presented previously (Tenhunen et al., $1976 \mathrm{a}, \mathrm{b}$ ) and continues to elaborate sub-processes in terms of physiologically meaningful parameters. The description is considered a working hypothesis. Data on photorespiration from the literature are reviewed as they relate to this working hypothesis. Several lines of investigation are thereby suggested that will help clarify the role of photorespiration in whole leaf photosynthesis and determine the over-all utility of this modeling approach.
\end{abstract}

\section{Introduction}

The following description of the simultaneous processes of photosynthesis and photorespiration in a single leaf has been developed to serve as a working hypothesis in further investigations of the carbon dioxide and oxygen dependencies of net photosynthesis in $\mathrm{C}_{3}$ plants. A successful description of the over-all photosynthetic process must be consistent with observations summarized by Chartier and Prioul (1976). The approach to over-all photosynthesis modelling of $\mathrm{C}_{3}$ plants separates light and temperature effects from those of carbon dioxide and oxygen (Tenhunen et al., 1976a). At a single light intensity and leaf temperature, a successful description should provide or account for the following (Chartier and Prioul, 1976):

(1) Saturation of net photosynthesis and suppression of oxygen inhibition at high $\mathrm{CO}_{2}$ concentration.

(2) A decrease in photosynthesis and an increase in photorespiration as 
oxygen concentration is increased above 1\% (Ludwig and Canvin, 1971; Tregunna et al., 1966; Forrester et al., 1966).

(3) A decrease in the initial slope of carbon dioxide response curves with increasing oxygen concentration in a manner described by Ogren and Bowes (1971) and Forrester et al. (1966).

(4) A linear increase of the $\mathrm{CO}_{2}$ compensation point with increasing oxygen concentration (Forrester et al., 1966).

(5) Estimates of $\mathrm{CO}_{2}$ evolution into $\mathrm{CO}_{2}$ free air compatible with those observed (Ludwig and Canvin, 1971; Yemm, 1969).

(6) Accurate prediction of the relationship of net photosynthesis rate as a function of carbon dioxide and oxygen concentrations.

(7) Possible physiological insight into the photosynthetic and photorespiratory processes of $\mathrm{C}_{3}$ plants and separation of these processes if possible.

The description must simulate responses of the type measured by Ludwig (private communication) for sunflower as shown in Figures 3 and 4 . The formulation of such a model has proven difficult because of the difficulty (Ludwig and Canvin, 1971; Schneider, 1969; Zelitch, 1966) of measuring the true photosynthetic response and photorespiratory response independently. The analysis presented here, based on considerations previously discussed by Lommen et al. (1971, 1975), Ogren and Bowes (1971), Laing et al. (1974), and Ogren (1975), performs well with respect to the criteria enumerated above. The analysis also performs well as an integral part of the more general description already mentioned in which light and temperature dependencies are included (Tenhunen et al., 1976a, b). Additional criteria concerning light and temperature phenomena fundamental to a successful model (Chartier and Prioul, 1976) as well as further criteria of our own are satisfied. This will be demonstrated in a following publication in which data of Joliffe and Tregunna (1973) are analyzed.

\section{Model Formulation}

The following considerations provide the basic framework for including photorespiration in our photosynthetic model for plants using the $\mathrm{C}_{3}$ mechanism of carbon fixation. Tenhunen et al. [1976a, Eq. (12)] suggested that at any fixed light intensity and leaf temperature and with photorespiration essentially zero, i.e. $\left[\mathrm{O}_{2}\right] \simeq 0$, the carbon dioxide $\left(\mathrm{C}_{A}\right)$ response of photosynthesis is explained in terms of a Michaelis constant for carbon dioxide $\left(K_{C}\right)$, a maximum rate for photosynthesis $\left(P_{M}\right)$, and a mesophyll diffusion resistance $\left(R_{M}\right)$. A temperature dependent residual respiration term [mitochondrial, see Eq. (5) of Tenhunen et al., 1976a] must also be included. When correction is made for residual respiration, the $\mathrm{CO}_{2}$ response curve is similar to curve a in Figure 1. As $\left[\mathrm{O}_{2}\right]$ is increased, net photosynthesis corrected for residual respiration decreases due to photorespiration and inhibition of $\mathrm{CO}_{2}$ fixation, and the response with respect to $\mathrm{CO}_{2}$ resembles curve $\mathrm{b}$ in Figure 1 . This oxygen dependent respiration and inhibition is described here based on ideas involving the interaction of $\mathrm{CO}_{2}, \mathrm{O}_{2}$ and $\mathrm{RuDP}$ carboxylase (Laing et al., 1974), and further including a diffusion path for $\mathrm{CO}_{2}$ (and diffusion resistance) from the intercellular air space to the chloroplast. 

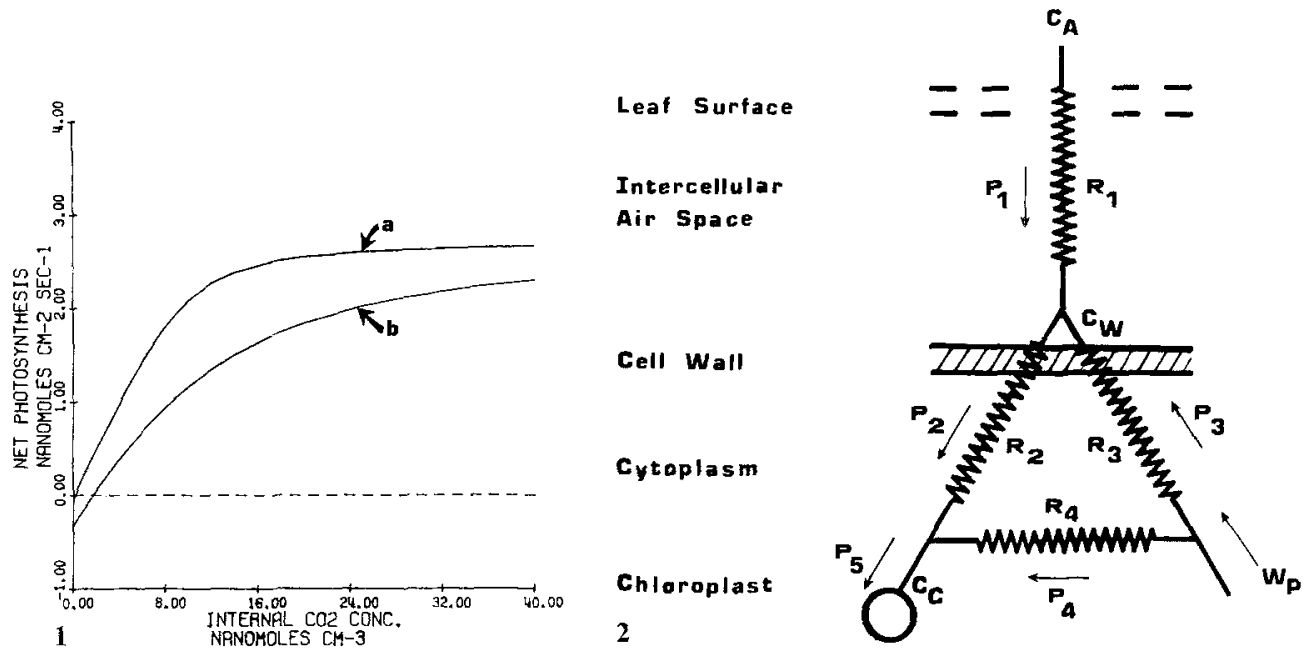

Fig. 1. Illustration of $\mathrm{CO}_{2}$ response described in the text. Curve $a$ observed at low oxygen concentration with photorespiration essentially zero. Curve $b$ observed at oxygen concentrations substantially above $1 \%$ with photorespiration occurring

Fig. 2. Electrical analogue of $\mathrm{CO}_{2}$ exchange in leaves including a photorespiratory source of $\mathrm{CO}_{2}$. Fluxes are taken as positive when they are in the directions indicated by the arrows. The $R^{\prime} \mathrm{s}$ are resistances, $P$ 's are $\mathrm{CO}_{2}$ fluxes, and $C^{\prime}$ s are $\mathrm{CO}_{2}$ concentrations (Lommen et al., 1971). It should be noted that $C_{W}$ in this figure has replaced $C_{1}$ in Lommen's terminology and $W_{p}$ replaces $W . C_{W}$ is the carbon dioxide concentration in the intercellular air spaces of the leaf at the cell walls of photosynthesizing leaf cells

Using the electrical analogue of $\mathrm{CO}_{2}$ exchange in the $\mathrm{C}_{3}$ leaf shown in Figure 2, Lommen et al. (1971) derived the following expression for net photosynthesis:

$$
\begin{aligned}
P= & \frac{\left[C_{A}+K_{C}+S_{1}\left(P_{M}-W_{P}\right)-W_{P} S_{2}\right]-\left\{\left[C_{A}+K_{C}+S_{1}\left(P_{M}-W_{P}\right)-W_{P} S_{2}\right]^{2}-4 S_{1}\left[\left(C_{A}-W_{P} S_{2}\right)\left(P_{M}-W_{P}\right)-W_{P} K_{C}\right]\right\}^{1 / 2}}{2 S_{1}} \\
S_{1} & \equiv R_{1}+\frac{R_{2}\left(R_{3}+R_{4}\right)}{R_{2}+R_{3}+R_{4}} \\
S_{2} & \equiv \frac{R_{2} R_{4}}{R_{2}+R_{3}+R_{4}}
\end{aligned}
$$

where: $P_{M}$ is the rate of photosynthesis at a specific light intensity, saturating carbon dioxide, and a specific leaf temperature $\left(\mathrm{nM} \mathrm{cm} \mathrm{cm}^{-2} \mathrm{~s}^{-1}\right)$, $W_{P}$ is the rate of photorespiration based on $\mathrm{CO}_{2}$ evolution $\left(\mathrm{nM} \mathrm{cm}^{-2} \mathrm{~s}^{-1}\right.$ ), $C_{A}$ is the concentration of carbon dioxide in air outside the boundary layer $\left(\mathrm{nM} \mathrm{cm}^{-3}\right)$,

$K_{C}$ is a constant equal to the chloroplast concentration of carbon dioxide (photorespiration $\simeq 0$ ) at which $P=P_{M} / 2$. 
If photorespiration $\left[W_{P}\right.$ in Eq. (1)] results from competition between $\mathrm{O}_{2}$ and $\mathrm{CO}_{2}$ for the enzyme ribulose diphosphate carboxylase (Tenhunen et al., 1976a, p. 91; Ogren, 1975; Chollet and Ogren, 1975), the Michaelis-Menten constant $K_{\mathrm{C}}$ in the above equation can only be found and only holds when $\left[\mathrm{O}_{2}\right] \simeq 0$. If $\left[\mathrm{O}_{2}\right]>0$ a competitive situation occurs where the apparent $K_{C}$ is a function of oxygen concentration. If $\beta=\left(1+\frac{\left[\mathrm{O}_{2}\right]}{K_{\mathbf{O}_{2}}}\right)$, then $K_{C} \beta$ replaces $K_{C}$ in Equation (1) where $K_{\mathrm{O}_{2}}$ is the oxygen concentration that results in a doubling of $K_{C}$.

If photorespiration is controlled at ribulose diphosphate carboxylase, then it is described in Equation (4) in terms of the same parameters (Ogren, 1975) and is competitively inhibited by chloroplast carbon dioxide concentration.

$$
W_{p}=\frac{W_{M}}{1+\frac{K_{\mathrm{O}_{2}}\left(1+\frac{C_{c}}{K_{\mathrm{C}}}\right)}{\left[\mathrm{O}_{2}\right]}}
$$

where: $W_{M}$ is the rate of photorespiration based on $\mathrm{CO}_{2}$ evolution at saturating oxygen, a specific leaf temperature, and a specific light intensity (nM cm ${ }^{-2} \mathrm{~s}^{-1}$ ),

$K_{\mathrm{O}_{2}}$ is a constant equal to the oxygen concentration $\left(C_{c} \simeq 0\right)$ at which $W_{P}=W_{M} / 2$ as a decimal fraction $(V / V)$,

$\left[\mathrm{O}_{2}\right]$ is the concentration of oxygen in the air as a decimal fraction $(\mathrm{V} / \mathrm{V})$, $C_{c}$ is the concentration of carbon dioxide at the chloroplast $\left(\mathrm{nM} \mathrm{cm}^{-3}\right)$.

In this relationship we note that photorespiration is essentially zero if $\left[\mathrm{O}_{2}\right]$ is very low or if $C_{c}$ is very high.

If $R_{1}$ (Fig. 2) is very low, then $C_{w}=C_{A}, S_{1} \equiv \frac{R_{2}\left(R_{3}+R_{4}\right)}{\left(R_{2}+R_{3}+R_{4}\right)}$, and Equation (1) applies to net photosynthetic responses with respect to carbon dioxide concentration in the intercellular air space $\left(C_{w}\right)$ which can be calculated from water exchange data. Problems which would otherwise be caused by stomatal resistance changes thereby are avoided. There is no way at present to obtain directly values of $R_{2}, R_{3}$, and $R_{4}$. This problem is simplified by assuming that the photorespiratory $\mathrm{CO}_{2}$ production site is close to the chloroplast. $R_{2}$ is then $\simeq R_{3}$, and $R_{4}$ is some small fraction of $R_{2}$, i.e. $R_{2} / M$. Substituting these equalities with $R_{1}=0$ into Equations (2) and (3), $S_{1}=R_{2} \frac{(M+1)}{(2 M+1)}$ and $S_{1}=\left(S_{2}+M S_{2}\right)=(1+M) S_{2}$. Further requiring $M$ to be very large places the site of $\mathrm{CO}_{2}$ evolution in photorespiration close to the site of $\mathrm{CO}_{2}$ fixation. $S_{1}$ then approaches as a limit $R_{2} / 2$, which is in fact the overall mesophyll resistance determined when $\left[\mathrm{O}_{2}\right] \simeq 0$ [Tenhunen et al., 1976a; Eq. (12)]. Further, $S_{2}=S_{1} /(1+M)=R_{M} /(1+M)$.

In summary, the response of net photosynthesis as a function of $\mathrm{CO}_{2}$ and $\mathrm{O}_{2}$ with light and leaf temperature constant is given by:

$$
P_{1}=P=\frac{A A-\left\{(A A)^{2}-B B\right\}^{1 / 2}}{2 R_{M}}
$$


where:

$$
\begin{aligned}
& A A=C_{w}+K_{C} \beta+R_{M}\left(P_{M}-W_{P}\right)-W_{P}\left(\frac{R_{M}}{1+M}\right) \\
& B B=4 R_{M}\left[\left(C_{w}-W_{P} \frac{R_{M}}{1+M}\right)\left(P_{M}-W_{P}\right)-W_{P} K_{C} \beta\right] \\
& \beta=\left(1+\frac{\left[\mathrm{O}_{2}\right]}{K_{\mathrm{O}_{2}}}\right) \\
& W_{P}=\frac{W_{M}\left[\mathrm{O}_{2}\right]}{\left[\mathrm{O}_{2}\right]+K_{\mathrm{O}_{2}}\left(1+\frac{C_{c}}{K_{c}}\right)}
\end{aligned}
$$

$M=$ some large number; for now 100 ,

$K_{C}, P_{M}$, and $R_{M}$ are obtained initially from analysis of the $\mathrm{CO}_{2}$ response curve at $1 \%$ oxygen,

$\mathrm{C}_{w}$ and $\left[\mathrm{O}_{2}\right]$ are known,

$C_{c}$ can be determined iteratively,

$\mathrm{K}_{\mathrm{O}_{2}}$ and $W_{M}$ are derived from the best fit solution of the equation to data where photosynthesis is a function of both $\mathrm{CO}_{2}$ and $\mathrm{O}_{2}$ as shown in the following section.

There are few data sets in the available literature from which it is possible to thoroughly test these equations describing $\mathrm{C}_{3}$ photosynthesis with concurrent photorespiration. Responses are required to internal air space carbon dioxide concentration at several oxygen concentrations with light and leaf temperature constant. The $1 \%$ oxygen (non-photorespiring) case is required to obtain initial estimates of $R_{M}, P_{M}$, and $K_{C}$ as will be discussed. Available data commonly lack the $1 \%$ case and have only been measured between $0-300$ or $0-600$ ppm $\mathrm{CO}_{2}$ internal (or more likely external), making determinations of $P_{M}$ difficult and obscuring the true shape of the response curves. Often data are uncorrected for changes in stomatal resistance or state nothing about stomatal effects. Finally, data are often presented on solid line graphs which provide little information about the actual observed values. Nevertheless, reported here are promising results obtained by applying our analysis to data from Helianthus annuus. The results are discussed with respect to the modeling criteria outlined above.

\section{Method of Analysis}

Unpublished data values for net photosynthesis in sunflower leaves (Helianthus annuus) at $25^{\circ} \mathrm{C}$ as a function of carbon dioxide and oxygen concentration were obtained from Ludwig (private communication). The data are those upon which a solid line graph previously presented by Ludwig (1972) was based. The observations are shown in Figure 3 as a function of carbon dioxide concentration external to the leaf at four oxygen concentrations and in Figure 4 as a function of calculated intercellular air space carbon dioxide concentration. If stomatal resistances in sunflower are unaffected by changes in $\mathrm{CO}_{2}$ and $\mathrm{O}_{2}$ concentrations as suggested by Ludwig and Canvin (1971), either data set is appropriate for analysis. The only difference expected in the results of the two analyses would be a larger value for resistance obtained from the data in Figure 3, since resistance 
would in this case represent the mesophyll resistance plus an added constant diffusion resistance of the stomata and boundary layer (therefore, simply labelled $R$ in Fig. 3). We analyzed both data sets for comparison. There is greater information content in the data shown in Figure 3 (less pooling of data). Since the data of Figure 4 are calculated, these values possibly contain additional error introduced in the calculation of resistance from water exchange data.

The data obtained from Ludwig were first corrected for residual respiration by extrapolating the $\mathrm{CO}_{2}$ response curves at $1 \%$ oxygen to zero carbon dioxide concentration (Tenhunen et al., $1976 \mathrm{a}$ ) and by adding the estimated respiration rate to all data values. The added residual respiration was $0.03 \mathrm{nM} \mathrm{cm} \mathrm{cm}^{-2} \mathrm{~s}^{-1}$ for the external $\mathrm{CO}_{2}$ data and $0.12 \mathrm{nM} \mathrm{cm} \mathrm{cm}^{-2} \mathrm{~s}^{-1}$ for the internal $\mathrm{CO}_{2}$ data. These corrections are included in the data shown in Figures 3 and 4 . The reason these estimates differ considerably is not apparent. We can expect this difference in correction to influence the final solution as well.

The corrected data were studied according to methods of non-linear least squares analysis (Draper and Smith, 1966). The main program was provided by the University of Michigan Statistical Laboratory and has been described previously (Tenhunen et al., $1976 \mathrm{~b}$ ). Three specific subroutines were required to supplement the main program. These subroutines solve for net photosynthesis at particular values of $\mathrm{CO}_{2}$ concentration and $\mathrm{O}_{2}$ concentration, and solve for partial derivatives of the function used for net photosynthesis in each case with respect to the parameters to be estimated. These subroutines are described briefly here. Detailed documentation is available from the authors for those wishing to adapt the analysis to their own data and their own non-linear analysis program.

Subroutine A solves the quadratic equation describing net photosynthesis when photorespiration is essentially zero [Tenhunen et al., 1976a; Eq. 12]. The solution is straightforward, since the only independent variable $\left(C_{w}\right)$ is measured directly. The partial derivatives of the quadratic photosynthesis equation with respect to $K_{C}, R_{M}$, and $P_{M}$ are needed in the main program and are calculated in this subroutine. The case described by these equations is a special case of the general case discussed in this publication. Oxygen dependencies fall out. It can be applied to the $\mathrm{CO}_{2}$ dependency of photosynthesis in $\mathrm{C}_{4}$ plants if $\mathrm{CO}_{2}$ is concentrated by the PEP carboxylation system as has been suggested (Ogren, 1975; Keck and Ogren, 1976) and, with a different meaning, it can be applied to the $\mathrm{CO}_{2}$ dependency in $\mathrm{C}_{3}$ plants when photorespiration is inhibited.

Subroutine $\mathrm{B}$ performs an iterative solution for net photosynthesis according to Equations (5)-(9) and Fick's Law. Essential in this solution is an iterative determination of the chloroplast $\mathrm{CO}_{2}$ concentration $\left(C_{\mathrm{c}}\right)$ which competitively interacts with oxygen in the chloroplast to determine the relative velocities of photosynthesis and photorespiration. Use of subroutine $B$ results in parameter estimates of the oxygen dependent parameters $K_{\mathrm{O}_{2}}$ and $W_{M}$ while $K_{C}, R_{M}$, and $P_{M}$ are held constant at values determined with subroutine A. Partial derivatives of Equation (5) with respect to $K_{\mathrm{O}_{2}}$ and $W_{M}$ are found.

Reasonably good estimates of $K_{C}, R_{M}, P_{M}, K_{\mathrm{O}_{2}}$, and $W_{M}$ are obtained with subroutines $A$ and $B$. A final solution on the basis of least squares is obtained with subroutine $C$. Subroutine $C$ is a generalized version of subroutine $B$. Iteration is performed with respect to all five parameters; $K_{C}, R_{M}, P_{M}, K_{\mathrm{O}_{2}}$, and $W_{M}$. The photosynthesis function is solved in the same manner as in subroutine $B$. The only addition to the subroutine is the solution for the partial derivatives of the function in Equation (5) with respect to the parameters $K_{C}, R_{M}$, and $P_{M}$. This three step determination of $K_{C}, R_{M}, P_{M}, K_{\mathrm{O}_{2}}$, and $W_{M}$ was carried out on the corrected data of Figures 3 and 4 . Final parameter values are influenced slightly by choice of the initial estimates to be used with subroutine $C$.

\section{Results}

The parameter values determined in the procedure described above are indicated in Figures 3 and 4. The solid lines in these figures were determined according to Equations (5)-(9), with the parameters set at the indicated values. Note that $K_{\mathrm{C}}$ and $K_{\mathrm{O}_{2}}$ are expressed in different units in keeping with common usage (see the captions to Figs. 3 and 4). One of the criteria mentioned above is well satisfied by the model. 


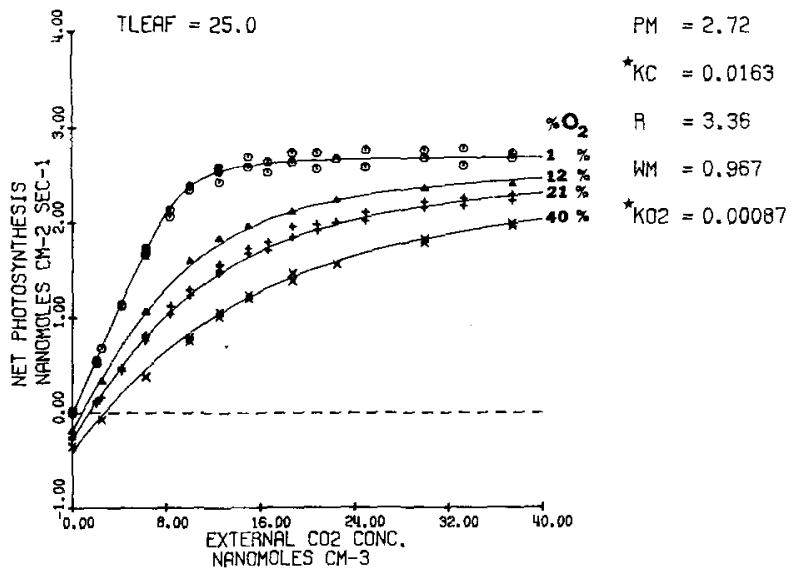

Fig. 3. Analysis of the sunflower data of Ludwig (1972) on the basis of external $\mathrm{CO}_{2}$ concentration $\left(C_{A}\right)$ according to the equations presented in the text. Symbols are Ludwig's observed values at $1,12,21$, and $40 \%$ oxygen. Solid lines are predicted according to Equations (5) through (9) with the parameter values shown. ${ }^{*}$ Note that $K_{C}$ and $K_{\mathrm{O}_{2}}$ are expressed in very different units in keeping with common usage. $K_{C}=0.0163 \mathrm{nM} \mathrm{cm}^{-3}$ carbon dioxide $=0.00004 \% ; K_{\mathrm{O}_{2}}=0.087 \%$ oxygen $(0.00087$ as a decimal fraction $\mathrm{V} / \mathrm{V})=35.6 \mathrm{nM} \mathrm{cm}^{-3}$; i.e. $K_{\mathrm{O}_{2}} \simeq 2,200 K_{\mathrm{C}} . R$ is the sum of mesophyll plus stomatal and boundary layer resistances in $\mathrm{s} \mathrm{cm}-1 . P_{M}$ and $W_{M}$ in $\mathrm{nM} \mathrm{cm} \mathrm{cm}^{-2}$ where $W_{M}$ is based on $\mathrm{CO}_{2}$ evolution. Light intensity $=116 \mathrm{~W} \mathrm{~m}^{-2} ; 400$ to $700 \mathrm{~nm}$

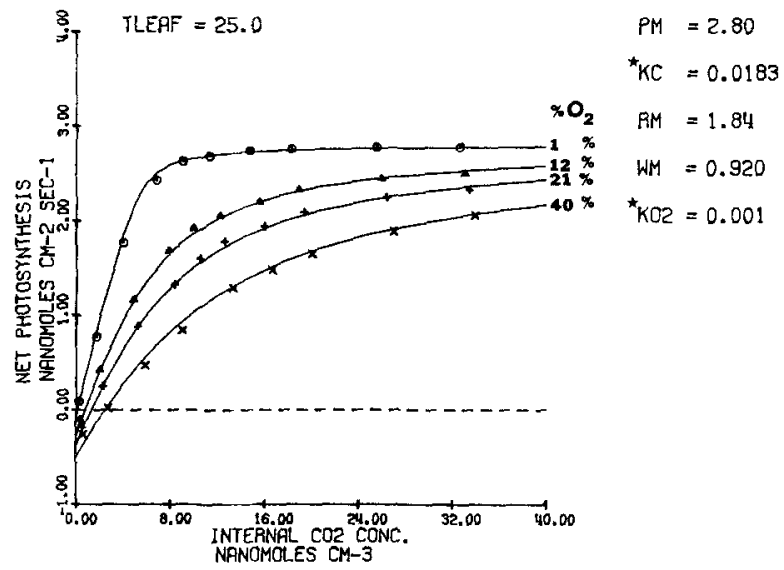

Fig. 4. Analysis of the sunflower data of Ludwig (1972) on the basis of internal $\mathrm{CO}_{2}$ concentration $\left(C_{W}\right)$ according to the equations presented in the text. Symbols are Ludwig's observed values at $1,12,21$, and $40 \%$ oxygen. Solid lines are predicted according to Equations (5)-(9) with the parameter values shown. ${ }^{*}$ Note that $K_{C}$ and $K_{\mathrm{O}_{2}}$ are expressed in very different units in keeping with common usage. $K_{C}=0.0183 \mathrm{nM} \mathrm{cm}^{-3}$ carbon dioxide $=0.000045 \% . K_{\mathrm{O}_{2}}=0.1 \%$ oxygen $(0.001$ as a decimal fraction $\mathrm{V} / \mathrm{V})=40.9 \mathrm{nM} \mathrm{cm}^{-3}$; i.e. $K_{\mathrm{O}_{2}} \simeq 2,200 K_{C} . R_{M}$ is the mesophyll resistance in $\mathrm{sm}^{-1}$. $P_{M}$ and $W_{M}$ in $\mathrm{nM} \mathrm{cm} \mathrm{cm}^{-2} \mathrm{~s}^{-1}$ where $W_{M}$ is based on $\mathrm{CO}_{2}$ evolution. Light intensity $=116 \mathrm{~W} \mathrm{~m}^{-2}$; 400 to $700 \mathrm{~nm}$ 
Accurate prediction is made of net photosynthesis rate as a function of carbon dioxide and oxygen concentration. The fit is better for the data in Figure 3 than in Figure 4. This may be the result of the larger number of observations considered in Figure 3 or may be the result of equal weighting of observations, with very few observations on the initial slope of the $\mathrm{CO}_{2}$ dependency at $1 \%$ oxygen in Figure 4 . The latter would tend to place less importance on goodness of fit in that region of the $1 \%$ oxygen response. As a result, the estimate of mesophyll resistance, which largely governs the response at $1 \%$ oxygen and low carbon dioxide concentration (Jones and Slatyer, 1972) may be slightly in error. We can expect a shift in parameter values estimated from the two data sets due to the different number of observations considered. This is precisely what is seen, but the shift is very small and the consistency in predicted values is more significant. Further results are presented only for the analysis based on data of Figure 3. Bear in mind that the resistance in this case is not only the mesophyll resistance, estimated from the results in Figure 4 to be approximately $1.84 \mathrm{~s} \mathrm{~cm}^{-1}$, but also stomatal plus boundary layer resistance of approximately $1.52 \mathrm{~s} \mathrm{~cm}^{-1}$. Again, this is only possible when no changes in stomatal resistance occur over the ranges in $\mathrm{CO}_{2}$ and oxygen concentrations shown, which is the case according to Ludwig and Canvin (1971). This is also supported by the agreement of predicted parameter values shown in Figures 3 and 4.

An important assumption we have made is that the site of photorespiration is much closer to the site of fixation than are the intercellular air spaces. This is supported by observations of the intimate association of chloroplasts, peroxysomes, and mitochondria (Newcomb and Frederick, 1971) which are thought to be involved in photorespiratory $\mathrm{CO}_{2}$ evolution. As $M \rightarrow \infty$ in Equation (5), the sites become identical. Clearly $M=\infty$ is unrealistic but how one should determine a value for $M$ is uncertain. The value of $M$ must a least be large enough to justify the simplifications of the resistance network outlined. The problem was approached by varying the value of $M$ and observing its effect on the predicted parameter values and the error mean square (EMS) of the least squares solution. The results are presented in Table 1 for different values of $M$. The EMS decreases very slightly (better solution) as $M$ decreases, but there is very little change in the predicted parameter values. A similar study of the data in Figure 4 demonstrated an even more consistent solution for values of $M$ between 10 and 1,000. At this time we have decided arbitrarily

Table 1. Predicted parameter values and error mean square value for solutions to the external $\mathrm{CO}_{2}$ dependencies shown in Figure 3 and for different values of $M$. See the caption of Figure 3 for parameter units

\begin{tabular}{rllllll}
\hline$M$ & $P_{M}$ & $K_{C}$ & $R$ & $W_{M}$ & $K_{\mathrm{O}_{2}}$ & EMS \\
\hline 10 & 2.72 & 0.0192 & 3.36 & 0.916 & 0.00100 & 0.064311 \\
25 & 2.72 & 0.0173 & 3.36 & 0.949 & 0.00091 & 0.064498 \\
50 & 2.72 & 0.0166 & 3.36 & 0.961 & 0.00088 & 0.064563 \\
100 & 2.72 & 0.0163 & 3.36 & 0.967 & 0.00087 & 0.064596 \\
500 & 2.72 & 0.0262 & 3.34 & 0.971 & 0.00140 & 0.064653 \\
\hline
\end{tabular}


on the value of 100 for $M$ as a compromise value that supports theoretical assumptions and agrees with the consistent solution of these data sets.

The predicted values of $P_{M}, R_{M}$, and $W_{M}$ are not unreasonable. The only other estimate of $W_{M}$ of which we are aware is half as large (Peisker and Apel, 1971; cucumber at $25^{\circ} \mathrm{C}$ ). However, since by the method of Peisker and Apel, photorespiration is only apparent when strongly dependent on oxygen concentration and will be underestimated, their estimate of $W_{M}$ is in good agreement with the value obtained here. The values of $K_{C}$ and $K_{\mathrm{O}_{2}}$ are low and very intriguing. Whether these values truly reflect properties of ribulose diphosphate carboxylase depends on the correctness of the model and accuracy of the data. These can be judged, it seems, only when further data sets of this type have been obtained. Data of Joliffe and Tregunna (1973), which will be analyzed in a later publication possibly suggest a higher value for $K_{\mathrm{O}_{2}}$ in the range of 1 to $10 \%$ oxygen and for $K_{C}$ around 0.1 to $1 \mu \mathrm{M}$. However, these data of Joliffe and Tregunna (1973) are plagued with several difficulties that create problems in analysis and in interpretation of that analysis. The $21 \%$ oxygen responses parallel the $1 \%$ oxygen responses more closely than expected on the basis of comparison with other data. There is a great deal of noise in general and particularly near the photosynthesis plateau. Overlapping of measurements on this plateau at $1 \%$ and $21 \%$ oxygen causes severe problems. Stomatal resistance changes due to temperature or $\mathrm{CO}_{2}$ and oxygen concentration changes are unaccounted for and may be the major cause of the inconsistencies noted.

In future applications of the model, important questions concerning the magnitude of parameter values, e.g. $K_{\mathrm{O}_{2}}$ and $K_{C}$, can only be resolved by obtaining consistent data over the entire range of responses. The data of Ludwig are consistent enough to demonstrate the usefulness of our approach and the characteristics of a system described by Equations (5)-(9). The results based on Ludwig's data are discussed below with respect to their possible import, in order to raise as many questions as possible pertaining to this area of research and to stimulate discussion over these attempts to draw together a broad knowledge of biochemistry, physiology, and ecology. It is our feeling that resolution of these questions and agreement on satisfactory values for parameters must await much further research.

The value of $K_{C}\left(0.0163 \mathrm{nM} \mathrm{cm}^{-3} \mathrm{CO}_{2} 0.00004 \% \mathrm{CO}_{2}\right.$ to half saturate photosynthesis) is extremely low when compared to the affinity of isolated ribulose diphosphate carboxylase for $\mathrm{CO}_{2}$. A value for $K_{C}$ one or two orders of magnitude larger is obtained as an initial estimate with subroutine $A$. This initial estimate is compatible with similar estimates on the same theoretical basis in cotton (Jones and Slatyer, 1972) and in bean (Tenhunen, unpublished), i.e. the 1\% oxygen response here is typical. The very low value of $K_{C}$ results during the final step of analysis with subroutine $C$. The ratio of $K_{\mathrm{O}_{2}}$ to $K_{C} \simeq 2200$ found here is in sharp contrast to the affinities reported for RuDP carboxylase by Laing et al. (1974) $\left(K_{\mathrm{O}_{2}} / K_{\mathrm{C}}=16\right.$ at $\left.25^{\circ} \mathrm{C}\right)$ and by Badger and Andrews (1974) $\left(K_{\mathrm{O}_{2}} / K_{\mathrm{C}} \simeq 10\right.$ at $\left.25^{\circ} \mathrm{C}\right)$.

The low value of $K_{C}$ is much smaller than previously reported for whole leaf photosynthesis as, for example, in cotton (Jones and Slatyer, 1972) at 
$1 \%$ oxygen $\left(K_{C}\right.$ as low as $\left.0.23 \mathrm{nM} \mathrm{cm}{ }^{-3} \mathrm{CO}_{2}=0.23 \mu \mathrm{M}\right)$. Bowes et al. (1975) report a $K_{C}$ for the carboxylase from freshly ruptured spinach chloroplasts of $7 \mu \mathrm{M}$. Bahr and Jensen (1974) report a similar value from spinach and tobacco. Bowes et al. (1975) and Bahr and Jensen (1974) indicate that these values are compatible with whole leaf photosynthesis. $7 \mu \mathrm{M}$ is 30 times greater than the value reported for cotton (Jones and Slatyer, 1972) and 430 times greater than the value reported here. We feel that this strongly underscores the importance to define clearly what is meant by apparent $K$ values, effective $K$ values, the leaf photosynthesis $K$, and $K_{m}\left(\mathrm{CO}_{2}\right)$ values reported. As shown here, the $K_{C}$ value is only as meaningful as the theoretical basis upon which it is derived. Since it is believed that no agreement on the proper expression for whole leaf photosynthesis has yet been reached, the enzymologist must be more cautious in finding agreement, or define more clearly what is in agreement and with which particular data, in order to force reexamination of data by those measuring whole leaf photosynthesis.

No success was had in significantly raising the predicted value of $K_{C}$ by using more complicated expressions of resistances in the model, e.g. using Equations (2) and (3) with low values of $M$, or by analyzing the uncorrected data and including residual respiration as a constant contribution to the photorespiratory flux. It would appear that a $K_{C}$ on the order of $1 \mu \mathrm{M}$ is incompatible with $C_{c}$ values determined solely on the basis of diffusion as in this model. One means of functioning with a higher $K_{C}$ is to have a $\mathrm{CO}_{2}$-concentrating mechanism (Gibbs et al., 1967). On the other hand, a slight shift $(5 \%)$ downward of the responses at 12,21 , and $40 \%$ oxygen will raise $K_{C}$ an order of magnitude to approximately $0.1 \mu \mathrm{M}$, underscoring the need for very precise measurement.

The $K_{\mathrm{O}_{2}}$ reported in this analysis is an order of magnitude lower than the $K_{\mathrm{O}_{2}}$ reported for the isolated ribulose diphosphate carboxylase enzyme. Estimates of the effective $K_{\mathrm{O}_{2}}$ in net photosynthesis have always been large (Chollet and Ogren, 1975). The effective $K_{\mathrm{O}_{2}}$ determined here is large not because of a large $K_{\mathrm{O}_{2}}$, but because of the ratio of $K_{\mathrm{O}_{2}} / K_{C}$ in the doubly competitive system. These values of $K_{\mathrm{O}_{2}}$ and $K_{C}$ if true imply that photorespiration is in fact considerable even with $\mathrm{O}_{2}$ concentration reduced to $1 \%$. Photorespiration rates for $1,12,21$, and $40 \%$ oxygen corresponding to the net photosynthesis responses shown in Figure 3 are given in Figure 5. As shown in the figure there is considerable photorespiration at low $\mathrm{CO}_{2}$, but it drops rapidly toward zero as external $\mathrm{CO}_{2}$ increases. At low $\mathrm{CO}_{2}$ concentration external, photosynthesis and photorespiration rapidly cycle within the leaf and net photosynthesis rate at zero $\mathrm{CO}_{2}$ external and $1 \%$ oxygen is essentially zero. This results because $C_{c}$ is still high (three times $K_{C}$ at zero $\mathrm{CO}_{2}$ external and $1 \%$ oxygen) due to photorespiration and diffusion resistance to the escape of $\mathrm{CO}_{2}$. The competitive situation rapidly swings in favor of carboxylation as $\mathrm{CO}_{2}$ external is increased resulting in high net photosynthesis rates. Chloroplast $\mathrm{CO}_{2}$ concentration curves corresponding to the responses in Figures 3 and 5 are given in Figure 6. If the description presented is proven to hold, the contention is supported (Samish and Koller, 1968; Zelitch, 1966) that photorespiration cannot be measured without specific inhibition of carboxylation $\left(P_{5}\right.$ in Fig. 2$)$ or photorespiration $\left(W_{P}\right)$. If $K_{\mathrm{O}_{2}}$ and $K_{C}$ are larger, a similar system is described 

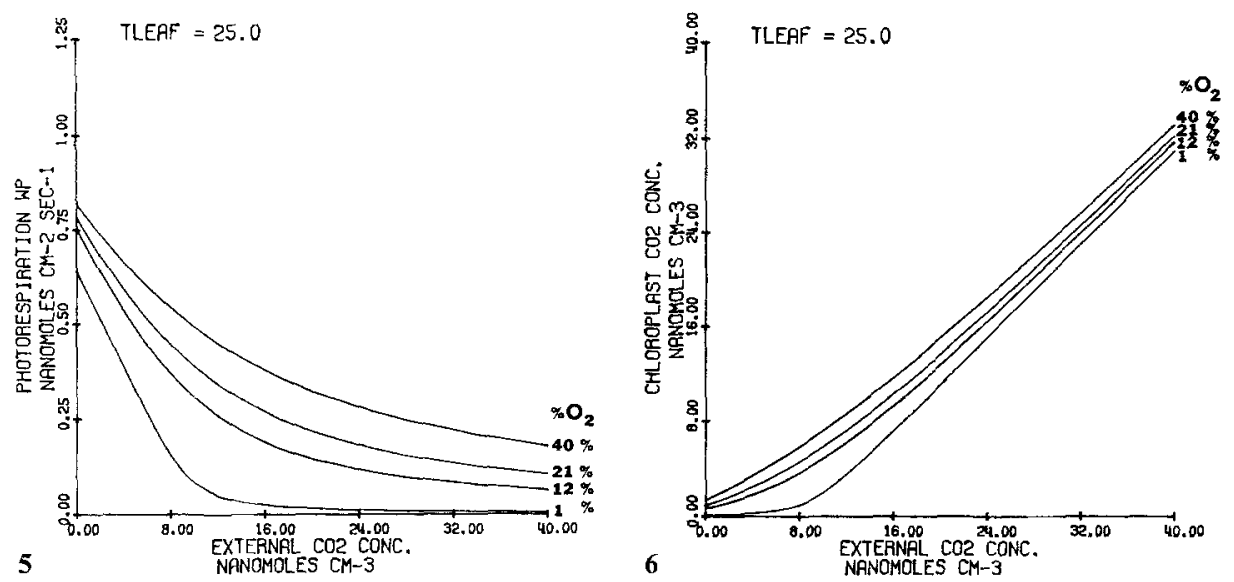

Fig. 5. Predicted relationship of photorespiration to external $\mathrm{CO}_{2}$ concentration at $1,12,21$, and $40 \%$ oxygen. This case corresponds to the solution shown in Figure 3 . See the caption of Figure 3 for parameter values used

Fig. 6. Predicted relationship of chloroplast $\mathrm{CO}_{2}$ concentration to external $\mathrm{CO}_{2}$ concentration at $1,12,21$, and $40 \%$ oxygen. This case corresponds to the solution shown in Figure 3 . See the caption of Figure 3 for parameter values

where the responses in Figures 3, 5, and 6 correspond to higher oxygen concentrations than those indicated.

An initial assumption in our analysis was that photorespiration was completely inhibited at $1 \%$ oxygen. Since it is not with these values of $K_{\mathrm{O}_{2}}$ and $K_{C}$, the assumptions upon which the analysis is based must be reexamined. $\mathrm{O}_{2}$ was reduced to $0.01 \%$ in simulation and the net photosynthesis response was predicted. The result is compared to the $1 \%$ oxygen response curve in Figure 7. One observes that even with these low $K$ values, photorespiration is essentially zero at $1 \%$ oxygen as far as might be seen from net photosynthesis responses. It would be extremely difficult to observe any differences in the initial slope (where $R_{M}$ can be determined) or on the plateau (where $P_{M}$ can be determined). The analysis demonstrates, however, that the oxygen dependency of photosynthesis in the curvature region may provide information on $K_{\mathrm{O}_{2}}$ and help in a critical evaluation of these data of Ludwig. Extrapolation of either curve would result in approximately the same value for residual respiration.

Since $K_{\mathrm{O}_{2}}$ is so low, small changes in oxygen concentration may be more important than originally assumed. As outlined by Samish (1975), the oxygen concentration at the photosynthesis site can only be approximately $0.03 \%$ greater than the external concentration. This is an upper estimate and the difference is probabiy less than half this. Since the $K_{\mathrm{O}_{2}}$ is $0.1 \%$ oxygen, the error due to the increase in oxygen at the photosynthesis site can be ignored, i.e. $1 \% /$ $K_{\mathrm{O}_{2}} \simeq 1.03 \% / K_{\mathrm{O}_{2}}$ as a competitive factor. This error is even less serious as oxygen concentration increases. Thus, the assumptions of the analysis are not seriously threatened by the unexpectedly low Michaelis constants obtained. 

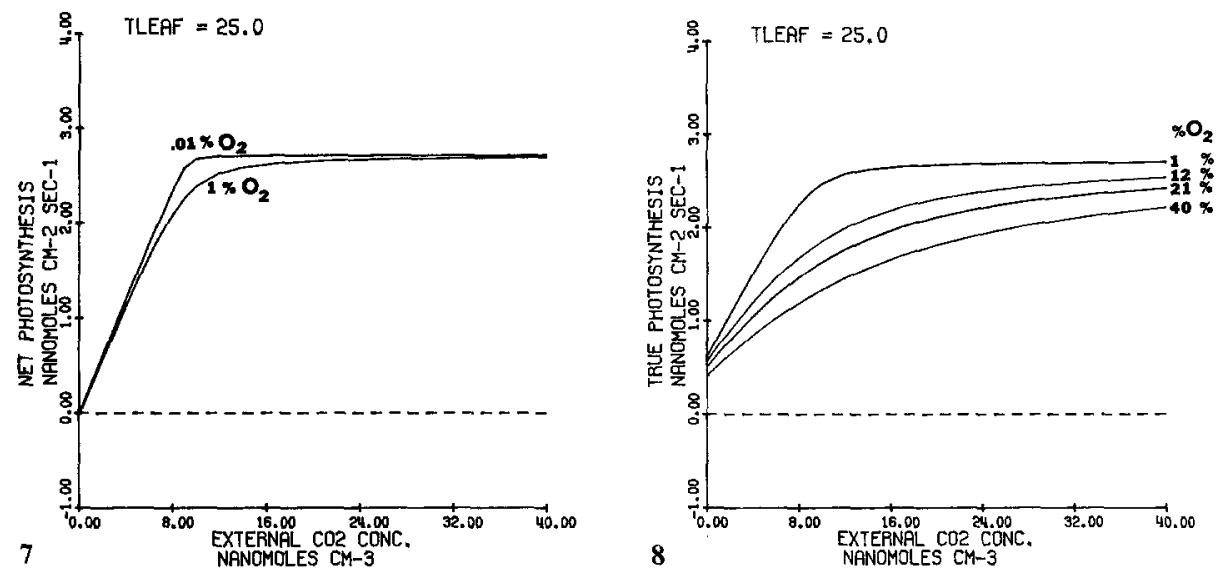

Fig. 7. Predicted comparison of the net photosynthesis response to external $\mathrm{CO}_{2}$ concentration at $0.01 \%$ (upper curve) and $1 \%$ (lower curve) oxygen. This case corresponds to the solution shown in Figure 3. See the caption of Figure 3 for parameter values

Fig. 8. Predicted relationship of 'true photosynthesis' to external $\mathrm{CO}_{2}$ concentration at $1,12,21$, and $40 \%$ oxygen. This case corresponds to the solution shown in Figure 3. See the caption of Figure 3 for parameter values

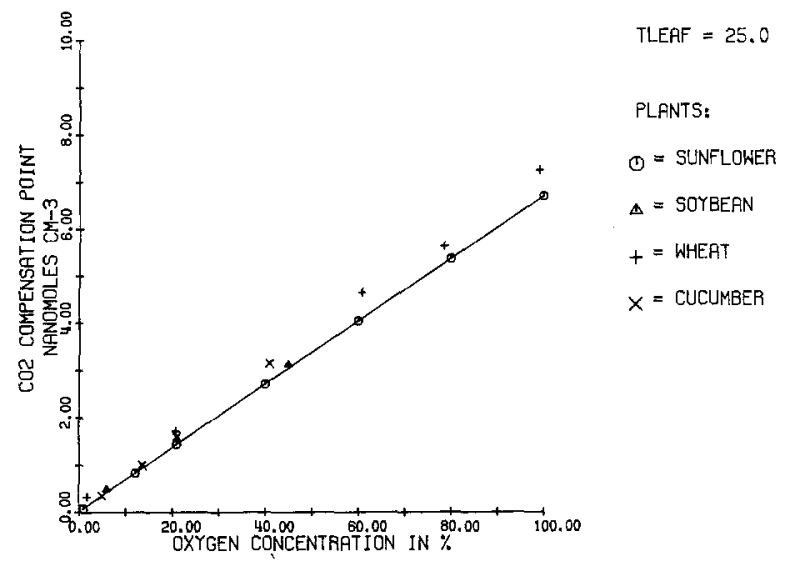

Fig. 9. The predicted as well as actual relationship of carbon dioxide compensation points to oxygen compensation according to the solution of Figure 3 (points joined by solid line). Measured dependencies of this relationship in soybean, wheat, and cucumber superimposed for comparison (symbols)

We can draw a picture of 'true photosynthesis' as a function of external $\mathrm{CO}_{2}$ concentration by adding net photosynthesis and photorespiration. This is shown in Figure 8 and corresponds to the data of Figures 3, 5, 6, and 7. It is questionable whether the term 'true photosynthesis' adds anything more than confusion to an already 'difficult topic. 'True photosynthesis' after all, may be only one aspect of the cycle through which the fluxes of photorespiration and net photosynthesis pass to regenerate substrate. We might consider referring to true carboxylation rate rather than 'true photosynthesis,' thus, remaining closer to the mechanism of interest and avoiding confusion with the many other aspects of photosynthesis now known.

It seems obvious from the fit of theory to the data in Figures 3 and 
4 that the predicted relationship of $\mathrm{CO}_{2}$ compensation point as a function of oxygen concentration will mimic reality. The prediction of this relationship according to the parameter values in Figure 3 is linear and is shown in Figure 9. This is discussed further below.

\section{Discussion and Conclusions}

The analysis described above suggests that the model formulated in the theory section of this paper may be the best description of $C_{3}$ photosynthesis to date. This is true if evaluation is based on model performance with respect to the criteria outlined in the introduction. Reviewing these criteria, as seen in Figures 3 and 5: (1) net photosynthesis saturates and photorespiration is suppressed at high carbon dioxide concentration due to competitive inhibition and the relative values of $K_{\mathrm{C}}$ and $K_{\mathrm{O}_{2}}\left(K_{\mathrm{O}_{2}}\right.$ ca. 2,200 times $\left.K_{\mathrm{C}}\right)$. (2) Increasing oxygen concentration affects photosynthesis in two ways; through inhibition of net photosynthesis by effecting a larger apparent $K_{C}$ and through increasing photorespiration by effecting a smaller apparent $K_{\mathrm{O}_{2}}$. (3) The initial slope of carbon dioxide response curves decreases with increasing oxygen concentration as discussed by Ogren and Bowes (1971). This is discussed in greater detail below. (4) A linear increase of $\mathrm{CO}_{2}$ compensation point with increasing oxygen concentration is an inherent part of the formulation as demonstrated in Figure 9. (5) Estimates of $\mathrm{CO}_{2}$ evolution into $\mathrm{CO}_{2}$ free air are obviously compatible with observation, since net photosynthesis data in figure 3 at zero carbon dioxide concentration fit well with theoretically predicted values. Net photosynthesis under these conditions is in fact $\mathrm{CO}_{2}$ evolution. As discussed below, these net photosynthesis data have not been found abnormal in any way when compared to data from a broad range of $\mathrm{C}_{3}$ plants. (6) Magnitudes of $K_{C}$ and $K_{\mathrm{O}_{2}}$ can be obtained for data as studied here and may be several orders of magnitude lower than has been previously suggested. This can only be determined when further experimental data have been gathered. Nevertheless, $K_{C}$ and $K_{\mathrm{O}_{2}}$ as used in this model may only be effective $K s$ and reflect the properties of a $\mathrm{CO}_{2}$-concentrating mechanism or further properties of this complex mechanism not specifically included. Photorespiration is revealed as a mechanism of rapid $\mathrm{CO}_{2}$ cycling even at zero net photosynthesis with zero carbon dioxide and $1 \%$ oxygen in the intercellular air space or external to the leaf. This is the result of $K_{\mathrm{O}_{2}}$ being several orders of magnitude greater than $K_{C}$ and a relatively high carbon dioxide $\left(C_{c}\right)$ concentration remaining in the leaf due to mesophyll resistance. These characteristics of the system hold even if $K_{\mathrm{O}_{2}} \simeq 1-10 \%$ oxygen and $K_{\mathrm{C}} \simeq 1 \mu \mathrm{M} \mathrm{CO}$ and are a result of the ratio of $K_{\mathrm{O}_{2}} / K_{C}$. These observations add support to hypotheses that photorespiration may provide a recycling mechanism to protect certain reaction centers when $\mathrm{CO}_{2}$ concentration is low.

Two unique aspects of this model are (1) that the effect of diffusion on the concentration of $\mathrm{CO}_{2}$ at the carboxylation site is included, with net photosynthesis rate a function of this determined value and (2) that the non-linear and saturation portion of response curves are included. The first of these with the 
iterative solution for net photosynthesis used in subroutines $B$ and $C$ overcomes a major difficulty that has been present in all other considerations of the problem to date. Including the non-linear and saturation portions of the response is significant, because it contributes to the unique determination of values for parameters describing this oxygen and carbon dioxide dependency. Including the saturation portion also allows linking of this analysis with the $P_{M}$ surface described previously (Tenhunen et al., 1976a). The solution to Ludwig's data demonstrates that the model provides accurate prediction of the relationship of net photosynthesis to carbon dioxide and oxygen. The results strongly support the advisability of modelling these dependencies on the basis of chloroplast $\mathrm{CO}_{2}$ concentration and the inadvisability of assuming, that in the linear portion of $\mathrm{CO}_{2}$ response curves, competition is negligible [Ogren, 1975; Eq. (7), $K_{\mathrm{O}} C$ is negligible] or that photorespiration is constant between $0-300 \mathrm{ppm} \mathrm{CO}_{2}$ (Samish and Koller, 1968; and others). This criticism is supported further by the observations of Yemm (1969) of $\mathrm{CO}_{2}$ release into $\mathrm{CO}_{2}$ free air dependent on steady-state $\mathrm{CO}_{2}$ and oxygen concentrations established before flushing with $\mathrm{CO}_{2}$ free air.

The results with this model are in sharp contrast to those of several other models with respect to the magnitude of $K$ values, magnitude of photorespiration, and relative magnitudes of mesophyll resistance and carboxylation resistance. In commenting on these, it is worthwhile to compare in detail this analysis with several other analyses (Jarvis, 1971). The work of Lake (1967a, b) has influenced almost all diffusion type photosynthesis models! This treatment differs from Lake's in three important aspects discussed below:

(1) Study of the resistance network of Figure 2 reveals that it is very similar to Lake's (1967a). The only difference is that we have removed a flux (Lake's $B_{i}$ ) representing respiration of non-photosynthesizing cells. It is felt that most of $B_{i}$ is accounted for in residual respiration (mitochondrial) corrections (Tenhunen et al., 1976a, b). It is our feeling that this treatment is more justified than trying to justify a specific respiratory flux into the intercellular air space. The approach has proven useful so far.

(2) All other differences between our model and Lake's are a result of the stepwise analysis outlined above. In our analysis, the resistance network is analytically expressed and then collapsed in such a way that the photorespiratory site is closely associated with the carboxylation site. Lake collapsed the network so that the photorespiratory site was moved to the cell wall. We feel that our approach is more reasonable as discussed in detail in the results section. Furthermore, it seems disadvantageous for photorespiratory $\mathrm{CO}_{2}$ to be dumped into the intercellular air spaces, a place where it is least likely to be reused.

(3) We have defined photorespiratory and photosynthesis functions and on the basis of these functions iteratively obtained $C_{c}$, net photosynthesis rate, and photorespiration rate. $C_{c}$ is not assumed equal to zero and overestimate of $R_{M}$ is no longer a problem (Lake, 1967b; Jarvis, 1971).

Mesophyll resistance in our model is treated as a constant diffusion resistance at a particular light intensity and leaf temperature (unaffected by oxygen concentration) but may include non-diffusion processes as discussed by others (Prioul et a1., 1975; Jarvis, 1971). Carboxylation resistance (Jarvis, 1971; Chartier, 1970; Jones and Slatyer, 1972; Chartier and Prioul, 1976) is inversely propor- 
Fig. 10. Predicted relationship of carboxylation resistance $R_{x}$ to external $\mathrm{CO}_{2}$ concentration at $1,12,21$, and $40 \%$ oxygen over those ranges of $\mathrm{CO}_{2}$ concentration in which Equation (10) is interpretable. This case corresponds to the solution shown in Figure 3. See the caption in Figure 3 for parameter values

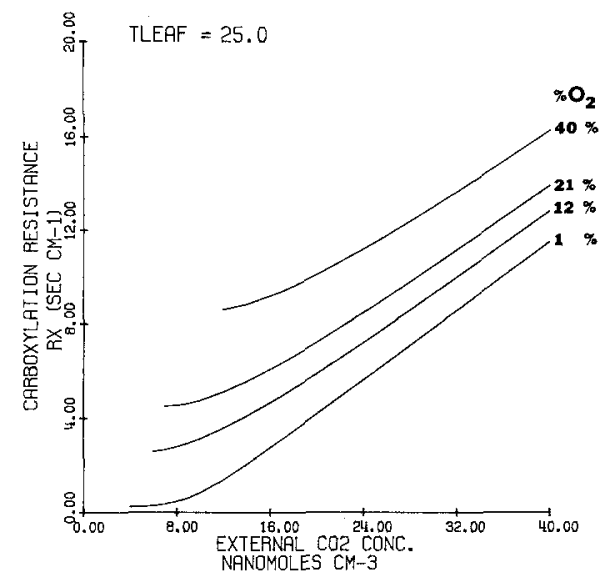

tional to the net photosynthesis rate and is defined as:

$$
R_{x}=\frac{C_{c}}{P}=\frac{C_{c}}{P_{5}-W_{P}}
$$

where: $P_{5}$ is the 'true photosynthesis' or true carboxylation rate $\left(\mathrm{nM} \mathrm{cm}{ }^{-2} \mathrm{~s}^{-1}\right)$.

As pointed out by Jarvis (1971), 'flow' across this resistance is quite different in comparison to other resistances. This concept of carboxylation resistance is perhaps most useful for comparisons at well defined concentrations of carbon dioxide and oxygen, since as $P \rightarrow 0$ and becomes negative, i.e. as photorespiratory fluxes dominate the relationship and finally exceed carboxylation, the relationship is uninterpretable. $R_{x}$ is dependent on photorespiration as indicated in Equation (10). The resulting $\mathrm{CO}_{2}$ and oxygen dependencies of the relationship are shown for the sunflower data studied here in Figure 10 in those ranges where we feel that it is meaningful.

At $P_{M}$ for a specific light intensity and leaf temperature, carboxylation resistance is the only resistance of significance and governs photosynthesis $\left(C_{c} \rightarrow \infty\right.$; $\left.P \rightarrow P_{M}\right)$. At less than saturation, $C_{c}$ and $P$ must be obtained in order to compare mesophyll resistance and carboxylation resistance. As Jarvis summarizes (1971, p. 579), net photosynthesis is strongly dependent on ambient $\mathrm{CO}_{2}$ at light saturation and $300 \mathrm{ppm} \mathrm{CO}_{2}$ but this only says that $R_{x}$ has decreased to some value small enough to make both $R_{x}$ and $R_{M}$ important.

In solving for $R_{x}$ and $R_{M}$ from light response curves, Chartier (1970) uses a model that combines light and carbon dioxide responses. As Prioul et al. (1975) report, the greatest error in their estimates of $R_{x}$ and $R_{M}$ may result from having chosen an improper description of the light response (Tenhunen et al., 1976a, b; Jassby and Platt, 1976). The choice of a particular light function will profoundly influence the curvature region of light response curves where data are taken for the Chartier analysis. Further assumptions made in their approach are that $R_{x}$ is constant and that error in estimating photorespiration has little affect on the results for these data. These assumptions must be reevaluated in the context of the discussion above.

Prioul et al. (1975) state that 'it is logical to compare $R_{x}$ variation with 
that of RuDP carboxylase (p. 19).' We feel that the comparison must be made with that of RuDP carboxylase-oxygenase, but only in carefully defined concentration ranges. With this interpretation and the definition shown in Equation (10), similarities and differences of $R_{x}$ in $\mathrm{C}_{3}$ and $\mathrm{C}_{4}$ plants are apparent. $\mathrm{C}_{4}$ carboxylation resistances are comparable to those of $\mathrm{C}_{3}$ plants at $1 \%$ oxygen $\left(1 \% \mathrm{O}_{2}\right.$ curves, Fig. 10). Net photosynthesis $(P)$ and carboxylation resistance $\left(R_{x}\right)$ are dependent on $C_{c}$ and $K_{C}$ [Tenhunen et al., 1976a, Eq. (12)], where $K_{C}$ is an effective $K$ that includes effects of the PEP carboxylation/concentration system (Ogren, 1975). In a $C_{3}$ plant, the effective $K$ is oxygen dependent $\left(K_{C} \beta\right)$. If $W_{P}$ is small enough, the $\mathrm{C}_{3}$ system responds similarly to the $\mathrm{C}_{4}$ system but real differences in interpretation between similar values of effective $K^{\prime \prime} \mathrm{s}$ must be kept in mind. At $21 \%$ oxygen, $C_{3}$ plants have higher total resistances than $C_{4}$ plants due in part to increase in $R_{x}$ (Fig. 10). While carboxylation resistance $\left(R_{x}\right)$ can be compared to mesophyll resistance, stomatal resistance, and boundary layer resistance, it is our opinion that limitations on the photosynthesis process are best understood on the basis of the photosynthesis function itself [Eq. (5)] because it includes carboxylation resistance and yet is meaningful at low carbon dioxide concentration.

The initial slopes of carbon dioxide curves result from the curvilinear dependency of $C_{c}$ on $C_{W}$ and the curvilinear dependency of net photosynthesis on $C_{c}$ [which is in contrast to the assumptions of Ogren (1975) and Samish and Koller (1968)]. They are not easily explained solely on the basis of adding mesophyll resistance to carboxylation resistance since carboxylation resistance is difficult to interpret at low carbon dioxide concentration. Nevertheless, the results here are not incompatible with ideas outlined by Peisker and Apel (1971) who recognized the contributions of carboxylation and photorespiratory fluxes to carboxylation resistance $\left(R_{x}\right)$. According to our analysis, photorespiration is not strongly dependent on oxygen concentration above $21 \%$ oxygen.

The results demonstrate the advantage of separating in analysis the effects of light, temperature, carbon dioxide, and oxygen. It is our feeling that lumping light, carbon dioxide, and oxygen can provide a predictive function (Chartier, 1970; Charles-Edwards and Ludwig, 1974; Hall, 1970), but limits one at this stage in our knowledge to a semi-empirical approach and may provide less physiological insight and possibly obscure basic assumptions.

In the presentation of results reported above, Ludwig's data were treated as 'good' data. We have compared these extensively with other data in the literature and found them to agree favorably with data of many authors from many $\mathrm{C}_{3}$ plants. The initial slope of carbon dioxide response curves ( $C E$ as used by Tregunna et al., 1966; Forrester et al., 1966; Ogren and Bowes, 1971) is a measure of gross conductance. If net photosynthesis is expressed in $\mathrm{nM} \mathrm{cm}{ }^{-2} \mathrm{~s}^{-1}$ and $\mathrm{CO}_{2}$ concentration in $\mathrm{nM} \mathrm{cm}^{-3}$, gross conductance $(C E$ for brevity) is expressed in $\mathrm{cm} \mathrm{s}^{-1}$. Regressions of $\ln (C E)$ as a function of oxygen concentration provide the following:

$$
\ln (C E)=-0.0183\left[\mathrm{O}_{2}\right]-1.259
$$

for Ludwig's data considering observations at 1, 12, 21 and $40 \% \mathrm{O}_{2}$;

$$
\ln (C E)=-0.0145\left[\mathrm{O}_{2}\right]-1.259
$$


for data of Laing et al. (1974) considering observations at 6, 21, and 45\% $\mathrm{O}_{2}$

where: $\left[\mathrm{O}_{2}\right]$ is expressed in $\%$ and $C E$ is in $\mathrm{cm} \mathrm{s}^{-1}$.

Consideration of this oxygen dependency leads to the conclusion that the sunflower leaves studied by Ludwig responded to oxygen in the linear portion of $\mathrm{CO}_{2}$ response curves in the same way as did soybean leaves studied by Laing et al. (1974). When oxygen is increased from $1 \%$ to $21 \%$, the initial slope at $21 \%$ oxygen is approximately 0.67 times the slope at $1 \%$ oxygen. This agrees with data from cucumber (Peisker and Apel, 1971), cotton (Lake and Slatyer, 1970), and several legumes (Ludlow, 1970). $C E_{0}$ (Ogren and Bowes, 1971 ) is the same for sunflower and soybean (=anti ln -1.259), but will change as will actual values of initial slopes when the resistances to diffusion change. For example, the corresponding relationship to Equation (10) above for the internal $\mathrm{CO}_{2}$ data of Figure 4 provides:

$$
\ln (C E)=-0.0264\left[\mathrm{O}_{2}\right]-0.731 ; C E_{0}=\operatorname{anti} \ln -0.731
$$

It is, thus, questionable whether $C E$ provides much insight as suggested by Ogren and Bowes (1971), even though it can clearly be used in a photosynthesis model such as they present.

The relationship of $\mathrm{CO}_{2}$ compensation point to oxygen concentration shown in Figure 9 is practically indistinguishable from that found in soybean at $25^{\circ}$ (Laing et al., 1974), in cucumber at $25^{\circ}$ (Peisker and Apel, 1971), and in wheat at $25^{\circ}$ (Joliffe and Tregunna, 1973). This comparison is shown in Figure 9 superimposed on the sunflower data. Such a comparison based on our model formulation further supports the contention of Laing et al. (1974) that RuDP carboxylase-oxygenase is the common factor controlling relative rates of photosynthesis and photorespiration in these plants.

In conclusion, upon closer inspection we have found no reason to question Ludwig's data. The only difference that we observe from other data sets is that the entire carbon dioxide response was obtained. As a result, the analysis outlined above can be applied. The only other data of the type needed in this analysis that we are aware of are those of Joliffe and Tregunna (1973) which will be discussed in a later paper. Critical evaluation of the model structure and the data base is needed from other workers and further data must be obtained in order to resolve the question whether the values of $K_{C}$ and $K_{\mathrm{O}_{2}}$ obtained here are realistic. Even if the magnitude of these parameter values is shown to be in error, the model presented here accomplishes two major goals. First, an accurate description of the net photosynthesis response is obtained and secondly, the effects of oxygen and carbon dioxide are separated. The characteristics of the sub-processes of carboxylation and photorespiration are likewise separated and change in either sub-process due to acclimation or adaptation (Tenhunen et al., 1976a) should be reflected in the parameter values determined in the analysis. That differences among plants may in the future be found is supported by Keck and Ogren (1976) who report a RuDP carboxylase with 'normal' affinity to $\mathrm{CO}_{2}$ and lowered affinity for oxygen in Panicum milioides. 
The formulation discussed here completes an initial version of a general physiological-ecological photosynthesis model that was discussed previously (Tenhunen et al., 1976a, b) and will be used as a working hypothesis. This general model has many considerations in common with models of Hall (1970), Chartier (1970), Charles-Edwards and Ludwig (1974), Laisk (1970), Lake (1967 a, b), and others, but differs in that it is developed specifically to separate the effects of light, temperature, carbon dioxide, and oxygen. It is this separation that provides its greatest advantage, namely a step-wise solution for physiologically meaningful parameters which regulate the rate of photosynthesis. It is clear from the formulation which data sets are required to describe net photosynthesis for a leaf as a function of light, temperature, carbon dioxide, and oxygen. These data may result in modifications and in inclusion of relationships defining interactions. Nevertheless, we feel that the basic model structure is sound and provides a coherent approach to modelling the photosynthesis function. It further provides the framework needed to study adaptation and acclimation of photosynthetic processes as discussed previously (Tenhunen et al., 1976a).

Acknowledgements. This research was supported by a grant from the Ford Foundation and by contract AT(11-1)-2164 from the Energy Research and Development Administration. We also wish to thank Meredy Gockel, Peter Harley, and Conrad Yocum for their contributions. In addition, we are particularly grateful to Dr. L.J. Ludwig for allowing us to use these unpublished data.

\section{References}

Badger, M., Andrews, T.: Effects of $\mathrm{CO}_{2}, \mathrm{O}_{2}$, and temperature on a high-affinity form of ribulose diphosphate carboxylase-oxygenase from spinach. Biochem. Biophys. Res. Comm. 60, 204-210 (1964)

Bahr, J., Jensen, R.: Ribulose diphosphate carboxylase from freshly ruptured spinach chloroplasts having an in vivo $K_{m}\left(\mathrm{CO}_{2}\right)$. Plant Physiol. 53, 39-44 (1974)

Bowes, G., Ogren, W., Hageman, R.: $\mathrm{pH}$ dependence of the $K_{m}\left(\mathrm{CO}_{2}\right)$ of ribulose 1,5-diphosphate carboxylase. Plant Physiol. 56, 630-633 (1975)

Charles-Edwards, D., Ludwig, L.: A model for leaf photosynthesis by $\mathrm{C}_{3}$ plant species. Ann. Bot. 38, 921-930 (1974)

Chartier, P.: A model of $\mathrm{CO}_{2}$ assimilation in the leaf. In: Prediction and measurement of photosynthetic productivity, pp. 307-315. Wageningen, The Netherlands: Pudoc 1970

Chartier, P., Prioul, J.: The effects of irradiance, carbon dioxide and oxygen on the net photosynthetic rate of the leaf: A mechanistic model. Photosynthetica 10, $20-24$ (1976)

Chollet, R., Ogren, W.: Regulation of photorespiration in $\mathrm{C}_{3}$ and $\mathrm{C}_{4}$ species. Bot. Review 41 , $137-179$ (1975)

Draper, N., Smith, H.: Applied regression analysis. New York: Wiley 1966

Forrester, M., Krotkov, G., Nelson, C.: Effect of oxygen on photosynthesis, photorespiration and respiration in detached leaves. I. Soybean. Plant Physiol. 41, 422-427 (1966)

Gibbs, M., Latzko, E., Everson, R., Cockburn, W.: Carbon metabolism: nature and formation of end products. In: Harvesting the sun: photosynthesis in plant life (A. San Pietro, F.Greer, T. Army, eds.), pp. 111-130. New York: Academic Press 1967

Hall, A.: Photosynthetic capabilities of healthy and beet yellow virus infected sugar beets (Beta vulgaris L.). Ph.D. Thesis, University of California, Davis (1970)

Jarvis, P.G.: The estimation of resistances to carbon dioxide transfer. In: Plant photosynthetic production, Manual of methods (Z. Sestak, J. Catsky, P. Jarvis, eds.), pp. 566-631. The Hague: Junk 1971

Jassby, A., Platt, T.: Mathematical formulation of the relationship between photosynthesis and light for phytoplankton. Limno. and Oceano. 21, 540-547 (1976)

Joliffe, P., Tregunna, E. : Environmental regulation of the oxygen effect on apparent photosynthesis in wheat. Can. J. Bot. 51, 841-853 (1973) 
Jones, H., Slatyer, R.: Estimation of the transport and carboxylation components of the intracellular limitation to leaf photosynthesis. Plant Physiol. 50, 283-288 (1972)

Keck, R., Ogren, W.: Differential oxygen response of photosynthesis in soybean and Panicum milioides. Plant Physiol. 58, 552-555 (1976)

Laing, W., Ogren, W., Hageman, R.: Regulation of soybean net photosynthetic $\mathrm{CO}_{2}$ fixation by the interaction of $\mathrm{CO}_{2}, \mathrm{O}_{2}$ and ribulose 1,5-diphosphate carboxylase. Plant Physiol. 54, $678-685$ (1974)

Laisk, A.: A model of leaf photosynthesis and photorespiration. In: Prediction and measurement of photosynthetic productivity, pp. 295-306. Wageningen, The Netherlands: Pudoc 1970

Lake, J.: Respiration of leaves during photosynthesis. I. Estimates from an electrical analogue. Aust. J. Biol. Sci. 20, 487-493 (1967a)

Lake, J.: Respiration of leaves during photosynthesis. II. Effects on the estimation of mesophyll resistance. Aust. J. Biol. Sci. 20, 495-499 (1967 b)

Lake, J., Slatyer, R. : Respiration of leaves during photosynthesis. III. Respiration rate and mesophyll resistance in turgid cotton leaves, with stomatal control eliminated. Aust. J. Biol. Sci. 23, $529-535$ (1970)

Lommen, P., Schwintzer, C., Yocum, C., Gates, D.: A model describing photosynthesis in terms of gas diffusion and enzyme kinetics. Planta (Berl.) 98, 195-220 (1971)

Lommen, P., Smith, S., Yocum, C., Gates, D.: Photosynthetic model. In: Perspectives of biophysical ecology (D. Gates, R. Schmerl, eds.), pp. 33-43. Berlin-Heidelberg-New York: Springer 1975

Ludlow, M.: Effect of oxygen concentration on leaf photosynthesis and resistances to carbon dioxide diffusion. Planta (Berl.) 91, 285-290 (1970)

Ludwig, L.: The relationship between photosynthesis and respiration. In: Crop processes in controlled environments (A.R. Rees, K.E. Cockshull, D.W. Hand, R.G. Hurd, eds.), pp. 305-326. London-New York: Academic Press 1972

Ludwig, L., Canvin, D.: The rate of photorespiration during photosynthesis and the relationship of the substrate of light respiration to the products of photosynthesis in sunflower leaves. Plant Physiol. 48, 712-719 (1971)

Newcomb, E., Frederick, S.: Distribution and structure of plant microbodies (peroxisomes). In: Photosynthesis and photorespiration (M.D. Hatch, C.B. Osmund, R.O. Slatyer, eds.), pp. 442-457. New York: Wiley 1971

Ogren, W.: Control of photorespiration in soybean and maize. In: Environmental and biological control of photosynthesis, pp. 45-52. The Hague: Junk 1975

Ogren, W., Bowes, G.: Ribulose diphosphate carboxylase regulates soybean photorespiration. Nature 230, 159-160 (1971)

Peisker, M., Apel, $P$.: Untersuchungen zum Einfluß von Sauerstoff auf den $\mathrm{CO}_{2}$-Gaswechsel assimilierender Blätter. Biochem. Physiol. Pflanzen (BPP) 162, 165-176 (1971)

Prioul, J., Reyss, A., Chartier, P.: Relationships between carbon dioxide transfer resistances and some physiological and anatomical features. In: Environmental and biological control of photosynthesis, pp. 17-28. The Hague: Junk 1975

Samish, Y.B.: Oxygen build-up in photosynthesizing leaves and canopy is small. Photosynthetica 9, 372-375 (1975)

Samish, Y., Koller, D.: Estimation of photorespiration of green plants and of their mesophyll resistance to $\mathrm{CO}_{2}$ uptake. Ann. Bot. 32, 687-694 (1968)

Schneider, K.: The Measurement of the true rate of photosynthesis with ${ }^{14} \mathrm{CO}_{2}$. Progress in Photosynthesis Research 1, 467-473 (1969)

Tenhunen, J., Yocum, C., Gates, D.: Development of a photosynthesis model with an emphasis on ecological applications. I. Theory. Oecologia (Berl.) 26, 89-100 (1976a)

Tenhunen, J., Weber, J., Yocum, C., Gates, D.: Development of a photosynthesis model with an emphasis on ecological applications. II. Analysis of a data set describing the $P_{M}$ Surface. Oecologia (Berl.) 26, 101-119 (1976b)

Tregunna, E., Krotkov, B., Nelson, C.: Effect of oxygen on the rate of photorespiration in detached tobacco leaves. Physiol. Plant. 19, 723-733 (1966)

Yemm, E.: Photorespiration and photosynthesis in young leaves of barley. Progress in Photosynthesis Research 1, 474-481 (1969)

Zelitch, I.: Increased rate of net photosynthetic carbon dioxide uptake caused by the inhibition of glycolate oxidase. Plant Physiol. 41, 1623-1631 (1966)

Received June 9, 1977 